
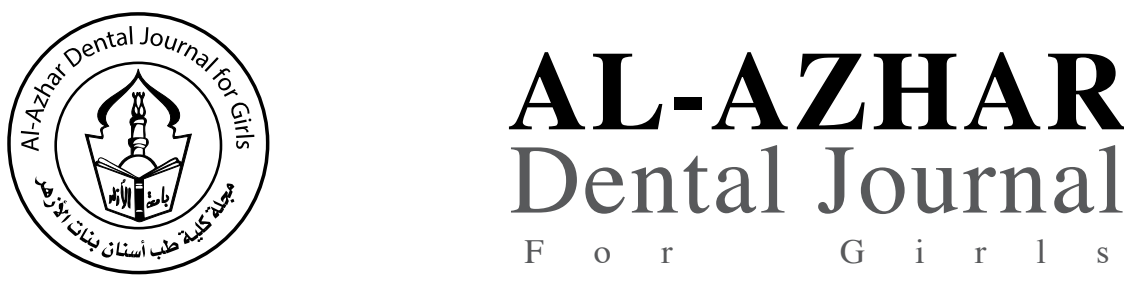

The Official Publication of The Faculty of Dental Medicine For Girls, Al-Azhar University Cairo, Egypt.

ADJ-for Grils, Vol. 4, No. 1, January (2017) — PP. 49:55

\title{
Effect of Smoking on the Expression of Human Beta-Defensin-2 in Gingival Crevicular Fluid after Non Surgical Periodontal Therapy
}

\author{
Riam Ahmed Saeed Binbarek ${ }^{(1)}$, Ossama Sayed A. El-Shall (2) and Mai Shafik Attia Mansour ${ }^{(3)}$
}

Codex : 07/1701

dentaljournal.forgirls@yahoo.com

\begin{abstract}
Aim: This study was designed to evaluate the effect of smoking on the expression of h $\beta \mathrm{D}-2$ in the GCF after non-surgical periodontal therapy. Subjects \& Methods: Ten non-smokers patients with chronic periodontitis and ten smoker patients with chronic periodontitis with age ranged between 25-40 years were selected for this study. All patients were examined with clinical periodontal parameters. Patients in both groups underwent nonsurgical periodontal therapy combined with a maintenance program (including brushing with regular toothpaste). GCF samples were collected all patients at baseline, one month as well as 3 months after periodontal therapy. Quantification of $\beta$-defensin-2 in human samples was measured using $\mathrm{h} \beta \mathrm{D}-2$ ELISA test. Results: Non-surgical periodontal therapy resulted in relative improvement in all clinical parameters as well as an increase in $\mathrm{h} \beta \mathrm{D}-2$ levels. In addition, GCF levels of $\mathrm{h} \beta \mathrm{D}-2$ were higher after non-surgical treatment in non-smoker groups than smokers. Conclusions: Deficiency of $\mathrm{h} \beta \mathrm{D}-2$ possibly could be related to host/microbial interaction and Smoking could modulate secretion of $h \beta D-2$, which represents a local defense dysfunction.
\end{abstract}

\section{INTRODUCTION}

Chronic periodontitis is the most prevalent form of periodontitis that is directly related to the longstanding plaque and calculus accumulation. However, environmental factors such as smoking may modify the host's immune response to the dental biofilm so that periodontal destruction becomes more progressive ${ }^{(1,2)}$.
Beta defensins-2; Chronic periodontitis; Smoking.
Paper extracted from Master Thesis entitled "Effect of Smoking on the Expression of Human Beta-Defensin-2 in Gingival Crevicular Fluid after Non Surgical Periodontal Therapy"

1. B.DS. 2010G Faculty of Dental Medicine University of Science and Technology -Sana'a-Yemen.

2. Professor and Chairman of Oral Medicine, Periodontology, Diagnosis and Radiology department, Faculty of Dental Medicine for Girls, Al-Azhar University.

3. Associate Professor of Oral Medicine, Periodontology, Diagnosis and Radiology department, Faculty of Dental Medicine for Girls, Al-Azhar University. 
Smoking is one of the main and most prevalent risk factors for chronic periodontitis. Smokers have demonstrated a decreased inflammatory response to plaque accumulation and reduced gingival bleeding $^{(3,4)}$. This altered inflammatory response has been attributed to alteration in the gingival vasculature which includes decreased vascular density, lumen area of gingival vessels, and epithelial thickness ${ }^{(5,6)}$. Furthermore, smoking produces a suppressive effect or impairment on various immune cells such as monocytes, neutrophils, lymphocytes and natural killer (NK) cells ${ }^{(7)}$. In addition, smoking diminishes the phagocytic uptake of both bacteria and apoptotic cells and induces qualitative and quantitative defects in circulating NK cells which are important in host viral and anti-tumor responses ${ }^{(8,9)}$. On the other hand, smoking also had a role in modulating the expression of pro-inflammatory cytokines in periodontal ligament and fibroblast cells ${ }^{(10)}$ as well as in oral keratinocytes and $\mathrm{GCF}^{(11-13)}$.

The goals of today's treatment of periodontitis are to reduce infection, resolve inflammation and create a clinical condition, which is compatible with periodontal health ${ }^{(14)}$. Non-surgical periodontal therapy consists of scaling and root planing (SRP) combined with oral hygiene instructions and their efficacy directly related to the ability of treatment to lower levels and prevalence of one or more pathogenic bacterial species. Typically, this results in attachment gain and pocket depth reduction due to a resolution of the inflammation ${ }^{(15-17)}$.

Antimicrobial peptides (AMPs) are multifunctional peptides whose fundamental biological role has been proposed to be the elimination of a diverse spectrum of microorganisms ${ }^{(18)}$. The most important antimicrobial peptide group in humans is defensins. Defensins have the ability to inactivate many bacteria, fungi, and some enveloped viruses. In humans, defensins can be subdivided into two families: alpha-defensins and beta-defensins ${ }^{(19,20)}$.

The human beta-defensins (h $\beta \mathrm{Ds}$ ) are small, cationic AMPs made primarily by epithelial cells and expressed in all human epithelia ${ }^{(21)}$. The $\mathrm{h} \beta \mathrm{Ds}$ are secreted in biological fluids, including urine, bronchial fluids, nasal secretions, saliva and gingival crevicular fluid ${ }^{(22-24)}$.

Among different AMPs, in the oral cavity, $\mathrm{h} \beta \mathrm{D}$ s2 was found to be 10 -fold more potent than $h \beta D-1$ and exhibited microbicidal activities against grampositive and gram-negative bacteria, fungi, and some parasites ${ }^{(25-27)}$. Furthermore, h $\beta \mathrm{D}-2$ mainly secreted in response to stimulation. This stimulation does not necessarily come only from bacteria since proinflammatory cytokines such as TNF- $\alpha$, interferon (IFN)-gamma, IL-1 $\beta$, IL-17, and IL-22, stimulate $\mathrm{h} \beta \mathrm{D}-2$ secretion. Otherwise, anti-inflammatory cytokines such as IL-4, and IL-10, suppress its production ${ }^{(28)}$. Besides, $\mathrm{h} \beta \mathrm{D}-2$ brings blood cells to the site of infection by acting as chemotactic agents $^{(28,29)}$. H $\beta D-2$ also was found to trigger fibroblast proliferation ${ }^{(30)}$. Additionally, $\mathrm{h} \beta \mathrm{D}-2$ has a strong impact on the maturation of premature osteoblasts which might be effective in bone tissue regeneration ${ }^{(31)}$. The aim of the present study was to investigate the effect of smoking on the expression of $\mathrm{h} \beta \mathrm{D}-2$ in the GCF after non-surgical periodontal therapy.

\section{SUBJECTS AND METHODS}

\section{Subjects:}

Twenty patients (age ranged 25-40 years) were selected from those attended to the Outpatient Clinics of Oral Medicine, Periodontology, Oral Diagnosis \& Radiology department, Faculty of Dental Medicine, Al-Azhar University (Girls' Branch), clinically diagnosed as having chronic periodontitis according to the classification of periodontal diseases by Armittage (1999) ${ }^{(32)}$. The criteria for inclusion in the current study were including patients free from any systemic conditions that affect periodontium or interfere with periodontal treatment, diagnosed as having moderate to advance chronic periodontitis ${ }^{(32)}$, did not receive any periodontal treatment in the past 
six months before the examination, and not receive antibiotics or anti-inflammatory therapy in the six months before the examination, for female patients, no pregnancy or lactation was included.

All individuals were informed about the procedures of the study and benefits of their participation in the study. A satisfactory written consent was obtained from all the patients denoting they're convinced about the schedule research program design. Ethical committee meeting approved the study protocol. The smoking history of the patients was evaluated using questionnaire, after which the patients were divided into two groups based on their smoking history. If the patient smoked more than 10 cigarettes per day, then he/she was classified as a smoker; if he/she had never smoked, then he/ she was classified as a non-smoker ${ }^{(33)}$.

Each patient's periodontal status was evaluated by measuring the Plaque Index (Pl) ${ }^{(34)}$, Gingival Index (GI) ${ }^{(35)}$ Probing Depth, Clinic Attachment Loss (CAL); at the baseline, 1-month, and at three months intervals by using Michigan 'O' Probe With Williams graduated periodontal probe. Ramfjord teeth $(16,21,24,36,41,44)$ or their substitutes were the target teeth for recording these parameters ${ }^{(36)}$.

\section{Collection of samples:}

Samples of gingival crevicular fluid were collected at baseline, one month and three months after periodontal therapy. The samples were pooled from four periodontal sites with attachment loss of $4 \mathrm{~mm}$ or more (in the four different quadrants). The sampling area was isolated with cotton rolls and carefully cleaned supragingivally with sterile cotton pellets. A sterile absorbent paper point was inserted into the gingival crevice or pocket until resistance was felt. The paper point was held in place for the 30s. The samples were immediately placed in Eppendorf tubes, transported to the laboratory and stored at $-80^{\circ} \mathrm{C}$. The collected samples analyzed using the enzyme-linked immunosorbent assay (ELISA) technique of human beta defensin-2 kit.

\section{Non-Surgical Periodontal Therapy:}

All patients in both groups were treated with nonsurgical periodontal therapy, which included the following: Supragingival and subgingival scaling and root debridement were performed with an ultrasonic device with iPiezo engine (NSK Varios 970, Japan), Chlorhexidine mouthwash was prescribed twice daily for one week post periodontal therapy and Oral hygiene instructions included brushing teeth with soft dental brush three times daily and using dental floss once a day.

\section{Quantification of Human beta defensin-2 using ELISA technique:}

Quantification of $\mathrm{h} \beta \mathrm{D}-2$ in human samples was measured using Bioneovan Inova h $\beta \mathrm{D}-2$ ELISA kit. The kit is suitable for testing a variety of sample types in-vitro and Purchased from Bioneovan Inova Co. Beijing, China. The kit assayed h $\beta D-2$ level in the sample, using a Purified $\mathrm{h} \beta \mathrm{D}-2$ antibody to coat microtiter plate wells, made a solid-phase antibody, then added h $\beta \mathrm{D}-2$ to wells, Combined HBD2 antibody which With HRP labeled, become antibodyantigen - enzyme-antibody complex. After washing Completely, Added TMB substrate solution, TMB substrate becomes blue color At HRP enzyme-catalyzed, reaction is terminated by the addition of a sulphuric acid solution and the color change is measured spectrophotometrically at a wavelength of $450 \mathrm{~nm}$. The concentration of $\mathrm{h} \beta \mathrm{D}-2$ in the samples is then determined by comparing the O.D. of the samples to the standard curve.

\section{Statistical analysis}

Values were presented as mean and standard deviation (SD) values. Data were explored for normality using Kolmogorov-Smirnov test of normality. The results of Kolmogorov-Smirnov test indicated that most of the data were normally distributed (parametric data), so one-way analysis of variance ANOVA test was used to compare between different intervals within the same group, followed 
by Tukey's post hoc test when the difference was found to be significant. Unpaired t-test was used to compare both groups (non-smokers, smokers). The significance level was set at $p \leq 0.05$. Statistical analysis was performed with SPSS 16.0 (Statistical Package for Scientific Studies, SPSS, Inc., Chicago, IL, USA) for Windows.

\section{RESULTS}

Table (1) showed the changes in the scores and measurements of PD and CLA from baseline, 1-month and 3-months after non-surgical periodontal therapy in the chronic periodontitis of smoker and non-smoker patients.

HßDs-2 ELISA analysis showed that in both groups, h $\beta \mathrm{D}-2$ level increased after non-surgical therapy, to reach the highest mean value after 3 months (Figure-1). One-way analysis of variance revealed that there is a statistically significant increase by time in both groups $(\mathrm{p}<0.0001)$. Tukey's post hoc test revealed no significant difference between mean values recorded at baseline (69.18 \pm 9.30$)$, after one month $(75.89 \pm 13.41)$ and after three months $(135.77 \pm 32.83)$ in the non-smokers group. However, in the smokers group, there was a significant difference between baseline $(61.99 \pm 12.97)$, after one-month $(79.90 \pm 13.33)$ and after three month observation times $(117.64 \pm 17.77)$.

At a baseline, a slightly greater mean $\mathrm{h} \beta \mathrm{D}-2$ level was recorded in non-smokers group (69.18 \pm 9.30$)$ than smoker group $(61.99 \pm 12.97)$, with no statistically significant difference $(\mathrm{p}=0.1714)$. At onemonth, a slightly greater mean $\mathrm{h} \beta \mathrm{D}-2$ level was recorded in smokers group (79.90+_13.33) than non-smokers $(75.89 \pm 13.41)$, with no statistically significant difference $(\mathrm{p}=0.0511)$. At three months, a slightly greater mean $\mathrm{h} \beta \mathrm{D}-2$ level was recorded in non-smokers group $(135.77 \pm 32.83)$ than smoker $(117.64 \pm 17.77)$ with no statistically significant difference $(\mathrm{p}=0.1420)$.

Table (1) Probing depth ( $\mathrm{mm})$ \& Clinical attachment level $(\mathrm{mm})$ in both groups.

\begin{tabular}{|c|c|c|c|c|c|c|c|}
\hline \multirow{2}{*}{ PD } & \multicolumn{2}{|c|}{ Baseline } & \multicolumn{3}{|c|}{1 month } & \multicolumn{2}{|c|}{3 months } \\
\hline & Non-smokers & Smokers & Non-smokers & \multicolumn{2}{|c|}{ Smokers } & Non-smokers & Smokers \\
\hline Mean & 3.17 & 3.33 & 2.55 & \multicolumn{2}{|c|}{3.01} & 1.84 & 2.53 \\
\hline SD & 0.50 & 0.48 & 0.52 & \multicolumn{2}{|c|}{0.42} & 0.54 & 0.51 \\
\hline $\mathrm{T}$ value & \multicolumn{2}{|c|}{1.7881} & \multicolumn{3}{|c|}{5.3306} & \multicolumn{2}{|c|}{7.1957} \\
\hline P value & \multicolumn{2}{|c|}{$0.0763^{\mathrm{ns}}$} & \multicolumn{3}{|c|}{$<0.0001 *$} & \multicolumn{2}{|c|}{$<0.0001 *$} \\
\hline \multirow{2}{*}{ CAL } & \multicolumn{2}{|c|}{ Baseline } & \multicolumn{3}{|c|}{1 month } & \multicolumn{2}{|c|}{ 3months } \\
\hline & Non-smokers & Smokers & Non-smokers & Smokers & Non-smokers & \multicolumn{2}{|c|}{ Smokers } \\
\hline Mean & 3.77 & 3.74 & 3.11 & 3.42 & 2.42 & \multicolumn{2}{|c|}{2.94} \\
\hline SD & 0.70 & 0.57 & 0.78 & 0.52 & 0.75 & \multicolumn{2}{|c|}{0.57} \\
\hline $\mathrm{T}$ value & \multicolumn{2}{|c|}{0.2574} & \multicolumn{2}{|c|}{2.5615} & \multicolumn{3}{|c|}{4.2758} \\
\hline$P$ value & \multicolumn{2}{|c|}{$0.7973^{\text {ns }}$} & \multicolumn{2}{|c|}{$0.0117 *$} & \multicolumn{3}{|c|}{$<0.0001 *$} \\
\hline
\end{tabular}




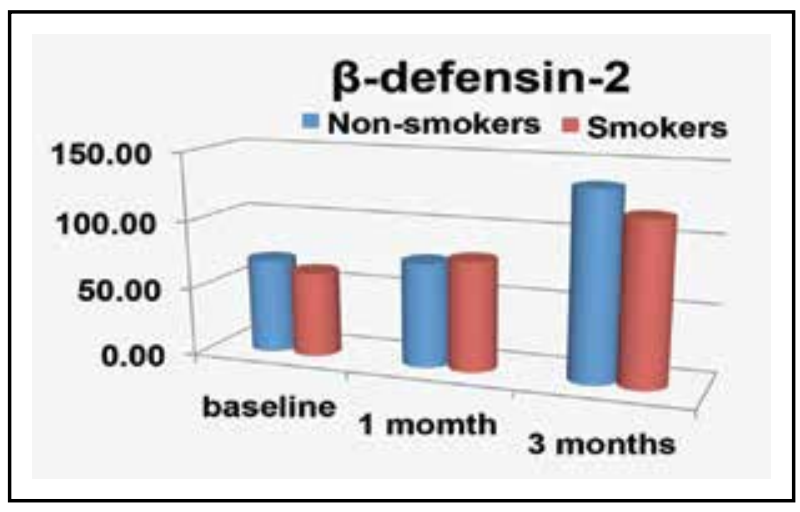

Fig. (1) Column chart showing mean h $\beta \mathrm{D}-2$ levels in both groups.

\section{DISCUSSION}

The complex effects of smoking on periodontal and oral diseases, and the mechanisms that mediate these diseases, are still considerably important. However, establishing a link between cigarette smoking and abnormal levels of antimicrobial peptides will provide a new insight into the epidemiology of the less favorable response following nonsurgical periodontal therapy.

Human beta defensin-2 considered one of the important AMPs in epithelial innate immunity, and their differential expression is associated with periodontal health and diseases. The h $\beta \mathrm{D}-2$ has a significant role as chemotactic, trigger fibroblast proliferation and has a strong impact on the maturation of premature osteoblasts, which might be effective in bone tissue regeneration. As such, the understanding of their role will undoubtedly unfold their clinical application in periodontal diseases.

According to Fan et al. (2015) ${ }^{(37)}$ the expression level of $\mathrm{h} \beta \mathrm{D}-2$ and $\mathrm{h} \beta \mathrm{D}-3$ in GCF among the smoking group was significantly lower than that in the non-smoking group. Also, The mRNA expression level of $h \beta D-2$ and $h \beta D-3$ in the smoking group was weakened compared with that in the non-smoking group indicating that smoking may have a negative effect on the immune defense system of the periodontal host, however, this study is in agreement with another study by Wang et $\boldsymbol{a l}$. (2015) ${ }^{(38)} \mathrm{dem}-$ onstrating that the whole cigarette smoke (WCS) exposure remarkably attenuated $\mathrm{h} \beta \mathrm{D}-1$ expression and secretion while clearly enhanced $h \beta D$ 2 and h $\beta \mathrm{D}-3$ expression levels, suggesting a link between cigarette smoke and abnormal levels of antimicrobial peptides.

The current study demonstrated that after nonsurgical periodontal therapy in both groups, $h \beta D-2$ level increased after non-surgical periodontal therapy, to reach the highest mean value after 3 months. However, at baseline, a slightly greater mean $\mathrm{h} \beta \mathrm{D}-2$ level was recorded in non-smokers group, with no statistically significant difference. At one-month, a slightly greater mean $\mathrm{h} \beta \mathrm{D}-2$ level was recorded in smokers group, with no statistically significant difference. At three months greater mean $\mathrm{h} \beta \mathrm{D}-2$ level was recorded in non-smokers group, with no statistically significant difference.

At baseline, the low level of h $\beta \mathrm{D}-2$ in smoker's group could be explained by the fact that periodontopathogenic microorganisms mainly P.gingivalis, which had a specific role in $\beta$-defensins degradation was found in smokers more than in non-smokers $(39,40)$. Moreover, bacteria with resistance to $\beta$-defensins, such as T. denticola and P.gingivalis, survive and colonize on epithelial surfaces, and eventually, invade gingival tissues ${ }^{(41)}$. With the bacterial invasion, $\beta$-defensins stimulate the secretion of chemokines, such as IL-8 and MCP-1, from dendritic cells, and, also, act as chemoattractants, which bring phagocytes and lymphocytes to the site of infection. Correspondingly, the activated immune response limits innate response and, hence, secretion of $\beta$-defensins ${ }^{(42)}$. Our findings were in agreement with previous studies shown that smoking downregulates $\mathrm{h} \beta \mathrm{D}-2$ expression ${ }^{(37,39,43)}$.

On the other hand, the proteolytic enzymes, which is produced by periodontal pathogens and the host in different ways such as (trypsin-like proteases and gingipains of P.gingivalis) potentially degrade and inactivate $\mathrm{h} \beta \mathrm{D}-2$ in-vitro conditions ${ }^{(44-47)}$. 
The improvement noticed in terms of clinical parameters after one and three months of non-surgical periodontal therapy when compared to baseline could be explained by the positive effect of nonsurgical periodontal therapy suggesting the relationship between host/bacterial factors of chronic periodontitis and $\mathrm{h} \beta \mathrm{D}-2$ levels in GCF.

In conclusion, the GCF levels of human $\mathrm{h} \beta \mathrm{D}$ 2 among chronic periodontitis patients could be changed depending on some factors such as smoking. Smoking might also affect the different clinical parameters including; PI, GI, PD and CAL. Moreover, the non-surgical periodontal therapy may lead to increased levels of $\mathrm{h} \beta \mathrm{D}-2$ in $\mathrm{GCF}$ among both smokers and non-smokers. However, The discrepancies of $\mathrm{h} \beta \mathrm{D}-2$ slight greater in smoker group after therapy, which could represents, a local defense dysfunction.

\section{REFERENCES}

1. Flemmig T F. Periodontitis. Annals of Periodontology, 1999;4: 32-7.

2. Stingu, Catalina Suzana, et al., Microbial profile of patients with periodontitis compared with healthy subjects. Quintessence International 2012; 43.

3. Rivera Hidalgo F. Smoking and periodontal disease. Periodontol 2000 2003;32:50-8.

4. Johnson $\mathrm{G} K$ and $M$ Hill. Cigarette smoking and the periodontal patient. J periodontol 2004;75:196-209.

5. BERGSTRöM J, L PERSSON and H. PREBER. Influence of cigarette smoking on vascular reaction during experimental gingivitis. European J Oral Sciences 1988;96:34-9.

6. Villa $\mathrm{C}$ and A Matorelli. Smoking influences on the thickness of marginal epithelium. Pesqui Odontol Bras 2003; 17:41-5.

7. Mehta H, K Nazzal and R Sadikot. Cigarette smoking and innate immunity. Inflammation Research 2008;57:497503.

8. Ginns L C, et al. Natural Killer Cell Activity in Cigarette Smokers and Asbestos Workers. American Review of Respiratory Disease1985;131:831-4.
9. Lu, Ling Min, et al. Cigarette smoke impairs NK celldependent tumor immune surveillance. J Immunology 2007;178:936-43.

10. Alpar, Berna, et al. Nicotine-induced alterations in human primary periodontal ligament and gingiva fibroblast cultures. Clinical oral invest 1998;2:40-6.

11. Petropoulos, Georgios, Ian J. McKay and Francis J. Hughes. The association between neutrophil numbers and interleukin- $1 \alpha$ concentrations in gingival crevicular fluid of smokers and non-smokers with periodontal disease. $\mathrm{J}$ clinical periodontol 2004;31:390-5.

12. Tymkiw, Keelen D, et al. Influence of smoking on gingival crevicular fluid cytokines in severe chronic periodontitis. $\mathrm{J}$ clinical periodontology 2011;38:219-28.

13. Johnson, Georgia K., et al. Interleukin-1 and interleukin-8 in nicotine-and lipopolysaccharide-exposed gingival keratinocyte cultures. J periodontal research, 2010; 45:583-8

14. Lang, Niklaus $\mathrm{P}$ and Maurizio S Tonetti. Periodontal risk assessment (PRA) for patients in supportive periodontal therapy (SPT). Oral Health Prev Dent 2003;1:7-16.

15. Stelzel, Michael and Lavin Florès de Jacoby. Topical metronidazole application as an adjunct to scaling and root planing. J clinical periodontol 2000;27:447-52.

16. Listgarten, M, J Lindhe and L Hellden, Effect of tetracycline and/or scaling on human periodontal disease. J Clinical Periodontol 1978;5;246-71.

17. Badersten, A, R Nilvéus and $\mathrm{J}$ Egelberg, Effect of nonsurgical periodontal therapy. $\mathrm{J}$ clinical periodontology 1981;8(1):57-72.

18. Diamond, Gill, et al. The roles of antimicrobial peptides in innate host defense. Current pharma design 2009; $15: 2377-92$

19. Froy, Oren. Regulation of mammalian defensin expression by Toll-like receptor-dependent and independent signalling pathways. Cellular microbio 2005;7:1387-97.

20. Scott, Monisha G and Robert EW Hancock. Hancock, Cationic antimicrobial peptides and their multifunctional role in the immune system. Criti Rev Immuno 2000;20.

21. Dale, Beverly A. Periodontal epithelium: a newly recognized role in health and disease. Periodontol 2000 2002;30;70-8.

22. Valore, Erika V, et al. Human beta-defensin-1: an antimicrobial peptide of urogenital tissues. J Clinical Investig 1998; 101:1633. 
23. Sahasrabudhe, $\mathrm{K}$, et al. Expression of the antimicrobial peptide, human $\beta$-defensin 1 , in duct cells of minor salivary glands and detection in saliva. $J$ dent research 2000;79:1669-74.

24. Diamond, Deborah L, et al. Detection of $\beta$-defensins secreted by human oral epithelial cells. J immuno methods 2001;256:65-76.

25. Yang, De, et al. Defensin participation in innate and adaptive immunity. Current pharma design 2007; 13:3131-39.

26. García, José Ramón Conejo, et al. Human $\beta$-defensin 4 a novel inducible peptide with a specific salt-sensitive spectrum of antimicrobial activity. FASEB J 2001; 15:1819-21.

27. Singh, Pradeep K, et al. Production of $\beta$-defensins by human airway epithelia. National Academy of Sciences 1998;95:14961-66.

28. Kanda, Naoko, et al. Human $\beta$-defensin- 2 enhances IFN- $\gamma$ and IL-10 production and suppresses IL-17 production in T cells. leukocyte bio 2011; 89:935-44.

29. Pazgier, $M$, et al. Human $\beta$-defensins. Cellular and Molecular Life Sciences 2006;63:1294-313.

30. Nishimura, Michiko, et al. Effect of defensin peptides on eukaryotic cells primary epithelial cells, fibroblasts and squamous cell carcinoma cell lines. J dermatological science 2004;36:87-95.

31. Kraus, D, et al. Human $\beta$-defensins differently affect proliferation, differentiation, and mineralization of osteoblast-like MG63 cells. J cellular physio 2012; 227:994-1003.

32. Armitage, Gary C. Development of a classification system for periodontal diseases and conditions. Annals of periodontol 1999; 4:1-6.

33. Ertugrul, A, et al. Gingival crevicular fluid levels of human beta-defensin-2 and cathelicidin in smoker and non-smoker patients a cross-sectional study. J periodontal research 2014; 49:282-9.

34. Silness, John, and Harald Löe. Periodontal disease in pregnancy II. Correlation between oral hygiene and periodontal condition. Acta odontologica scandinavica 1964; 22:121-35.

35. Löe, Harald, and John Silness. Periodontal disease in pregnancy I. Prevalence and severity. Acta odontologica scandinavica 1963; 21(6): p. 533-51.
36. Ramfjord, Sigurd P. Indices for prevalence and incidence of periodontal disease. J periodontology 1959; 30:51-9.

37. Fan, Y, et al. The impact of smoking on human beta defensin 2, 3 in gingival crevicular fluid and gingival tissue of patients with chronic periodontitis. Shanghai $\mathrm{j}$ stomatol 2015; 24:735-8.

38. Wang, Wen mei, et al. Effects of whole cigarette smoke on human beta defensins expression and secretion by oral mucosal epithelial cells. Tobacco induced diseases, 2015; 13:1-10.

39. Mahanonda, Rangsini, et al. Cigarette smoke extract modulates human $\beta$-defensin-2 and interleukin-8 expression in human gingival epithelial cells. J periodontal research 2009; 44:557-64.

40. Zambon, J J, et al. Cigarette Smoking Increases the Risk for Subgingival Infection With Periodontal Pathogens. J periodontol 1996; 67:1050-54.

41. Brissette, C, L Simonson, and S Lukehart. Resistance to human $\beta$-defensins is common among oral treponemes. Oral microbio and immunol 2004; 19:403-7.

42. Yin, Lei, et al. Differential and coordinated expression of defensins and cytokines by gingival epithelial cells and dendritic cells in response to oral bacteria. BMC immunol 2010; 11:37.

43. Wolgin, Michael, et al. HBD-1 and hBD-2 expression in HaCaT keratinocytes stimulated with nicotine. Oral biol 2012; 57:814-9.

44. Maisetta, Giuseppantonio, et al. Gingipains produced by Porphyromonas gingivalis degrade human- $\beta$-defensin 3 and affect peptide's antibacterial activity in vitro. Peptides 2011; 32:1073-7.

45. Kuula, Heidi, et al. Human $\beta$-defensin- 1 and- 2 and matrix metalloproteinase-25 and-26 expression in chronic and aggressive periodontitis and in peri-implantitis. Oral biol 2008; 53:175-86.

46. Brancatisano, F L, et al. Reduced human beta defensin 3 in individuals with periodontal disease. $\mathrm{J}$ dental research 2011; 90:241-5.

47. Taggart, Clifford $\mathrm{C}$, et al. Inactivation of human $\beta$-defensins 2 and 3 by elastolytic cathepsins. J Immunol 2003;171(2):931-7. 